\title{
ON WIENER PROCESS SAMPLE PATHS
}

BY

\author{
G. J. FOSCHINI AND R. K. MUELLER
}

\begin{abstract}
Let $\left\{X_{t}(\omega)\right\}$ represent a version of the Wiener process having almost surely continuous sample paths on $(-\infty, \infty)$ that vanish at zero. We present a theorem concerning the local nature of the sample paths. Almost surely the local behavior at each $t$ is of one of seven varieties thus inducing a partition of $(-\infty, \infty)$ into seven disjoint Borel sets of the second class. The process $\left\{X_{t}(\omega)\right\}$ can be modified so that almost surely the sample paths are everywhere locally recurrent.
\end{abstract}

We begin by illustrating our terminology. A function $f(t)$ is said to properly increase from the left at $t_{0}$ if there exists a $\delta>0$ such that $f(t)-f\left(t_{0}\right)<0$ for all $t \in\left(t_{0}-\delta, t_{0}\right)$. A function $f(t)$ is said to oscillate from the left at $t_{0}$ if for all $\delta>0$, $f(t)-f\left(t_{0}\right)$ takes both positive and negative values in $\left(t_{0}-\delta, t_{0}\right)$. Similar notions like points of improper increase, proper decrease from the right, oscillation from the right and bilateral oscillation have the obvious definitions.

THEOREM. Almost surely a sample path $X_{t}(\omega)$ admits the following exhaustive disjoint partition of $(-\infty,+\infty)$ :

$Q_{1}\left[X_{t}(\omega)\right]: A G_{\delta}$ of bilateral oscillation points which has complement of measure zero.

$Q_{2}\left[X_{t}(\omega)\right]:$ A countable dense set of proper local maxima.

$Q_{3}\left[X_{t}(\omega)\right]:$ A countable dense set of proper local minima.

The remaining sets are each $G_{\delta \sigma}$ 's (necessarily of measure zero) condensing about each $t \in(-\infty,+\infty)$ and of first category:

$Q_{4}\left[X_{t}(\omega)\right]$ : The points where $X_{t}(\omega)$ properly decreases from the left and oscillates from the right.

$Q_{5}\left[X_{t}(\omega)\right]$ : The points where $X_{t}(\omega)$ properly increases from the left and oscillates from the right.

$Q_{6}\left[X_{t}(\omega)\right]$ : The points where $X_{t}(\omega)$ properly decreases from the right and oscillates from the left.

$Q_{7}\left[X_{t}(\omega)\right]:$ The points where $X_{t}(\omega)$ properly increases from the right and oscillates from the left.

Presented to the Society, April 5, 1969; received by the editors February 26, 1969.

AMS Subject Classifications. Primary 6062; Secondary 2601.

Key Words and Phrases. Wiener process, sample paths, local behavior, Borel sets, oscillation from the right, increase from the left, local maxima, everywhere locally recurrent functions. 
The result that almost surely the set of points of maximum is countable contradicts the following two statements in [1]:

...the set of points of maximum is, almost surely, of the power of the continuum in every nonempty open interval.

... the set of values at the points of maximum though, almost surely, of the power of the continuum....

(These contradictions in no way affect the validity of the remarkable main result in [1].)

Proof of Theorem. Evidently $\left\{-X_{t}(\omega)\right\},\left\{X_{-t}(\omega)\right\}$, and $\left\{-X_{-t}(\omega)\right\}$ are also Wiener processes. This symmetry will prove useful in what follows.

Doob [2] shows that for any fixed $t$ almost surely $D^{+} X_{t}(\omega)=+\infty$. Thus by Fubini's theorem almost surely $\left\{t \mid D^{+} X_{t}(\omega)=+\infty\right\}$ has complement of measure zero. Using the symmetry it follows that almost surely $K=\left\{t: D^{+} X_{t}(\omega)=+\infty\right.$, $\left.D_{+} X_{t}(\omega)=-\infty, D^{-} X_{t}(\omega)=\infty, D_{-} X_{t}(\omega)=-\infty\right\}$ has complement of measure zero. Since $Q_{1}\left[X_{t}(\omega)\right] \supset K$ we see that $Q_{1}\left[X_{t}(\omega)\right]$ has complement of measure zero. We leave the proof that $Q_{1}\left[X_{t}(\omega)\right]$ is a $G_{\delta}$ for later.

Since $X_{t}(\omega)$ is almost surely monotone on no interval, the local maxima (local minima) of $X_{t}(\omega)$ are almost surely dense in $(-\infty,+\infty)$. Now almost surely all local maxima (local minima) are proper. This follows from the following lemma for which we are indebted to L. A. Shepp (private communication).

LEMMA. Almost surely the images of local maxima of $X_{t}(\omega)$ are distinct.

Proof. Let $P$ denote Wiener measure. Define the random variable $v\left(X_{t}(\omega), I\right)$ as the maximum value of $X_{t}(\omega)$ on the closed interval $I$.

If $I \cap J=\varnothing$ it is sufficient to show $P\left\{\omega \mid v\left(X_{t}(\omega), I\right)=v\left(X_{t}(\omega), J\right)\right\}=0$ since $\{\omega \mid$ two local maxima have the same ordinate $\} \subset \bigcup_{I, J}\left\{\omega \mid v\left(X_{t}(\omega), I\right)=v\left(X_{t}(\omega), J\right)\right\}$ where the union is taken over all pairs of disjoint closed intervals with rational endpoints. To prove $P\left\{\omega \mid v\left(X_{t}(\omega), I\right)=v\left(X_{t}(\omega), J\right)\right\}=0$ for $I \cap J=\varnothing$ suppose $I=[a, b]$ and $J=[c, d]$ for $a<b<c<d$. If $Y\left(X_{t}(\omega)\right)=v\left(X_{t}(\omega), I\right)$ and $Z\left(X_{t}(\omega)\right)=v\left(X_{t}(\omega), J\right)$, $P(Y=Z)=0$ since $(Y, Z)$ has a continuous distribution (absolutely continuous with respect to Lebesgue measure) [2].

Now the set of proper maxima of a continuous function is countable [3]. Thus we have shown that almost surely $Q_{2}\left[X_{t}(\omega)\right]$ and $Q_{3}\left[X_{t}(\omega)\right]$ are countable dense sets.

By symmetry we can prove the statements about $Q_{i}\left[X_{t}(\omega)\right], i=4,5,6,7$, by only considering $Q_{4}\left[X_{t}(\omega)\right]$. We limit our consideration to those $X_{t}(\omega)$ having dense enumerable points of proper maxima $\{m(j, \omega)\}_{j=0}^{\infty}$. It suffices to show $Q_{4}\left[X_{t}(\omega)\right]$ $=\bigcup_{j=0}^{\infty} T_{j}(\omega)$ where each $T_{j}(\omega)$ is a nowhere dense $G_{\delta}$ condensing at $m(j, \omega)$. We defer the definition of $T_{j}(\omega)$. Fix $j$ and $\omega$. To facilitate our explanation we relabel the $t$ axis so $m(j, \omega)=0$. Let $\mathscr{T}_{j}(\omega)=\left\{\tau \mid \tau \geqq 0, X_{t}(\omega) \geqq X_{\tau}(\omega)\right.$ for $\left.0 \leqq t \leqq \tau\right\}$. Except 
for $\tau=0$ each point of $\mathscr{T}_{f}(\omega)$ is such that $X_{t}(\omega)$ properly decreases from the left. Except for $\tau=0$ each point in $\mathscr{T}_{j}(\omega)$ is either a local minimum or a member of $Q_{4}\left[X_{t}(\omega)\right]$ since [1] shows that points of decrease almost surely do not occur. We demonstrate that $\mathscr{T}_{j}(\omega)$ is perfect. Associate with each $\tau \in \mathscr{T}_{j}(\omega)$ the planar line segment $\left[\left(0, X_{\tau}(\omega)\right),\left(\tau, X_{\tau}(\omega)\right)\right]$. To show that $\mathscr{T}_{j}(\omega)$ is closed we consider any Cauchy sequence $\left\{t_{n}\right\} \subset \mathscr{T}_{j}(\omega)$. The limit of the line segments associated with the $t_{n}$ is precisely the line segment required for $t$ to be in $\mathscr{T}_{j}(\omega)$. This is easily seen by considering the cases $t_{n} \uparrow$ and $t_{n} \downarrow$. So $\mathscr{T}_{j}(\omega)$ is closed. It remains to show that each point in $\mathscr{T}_{j}(\omega)$ is a limit point. If $\tau \in \mathscr{T}_{j}(\omega)$ is such that its associated open line segment does not meet the graph of $X_{t}(\omega)$ then $\tau$ is a limit point from the left of points in $\mathscr{T}_{j}(\omega)$. Suppose $\tau \in \mathscr{T}_{j}(\omega)$ is such that its associated open line segment meets the graph of $X_{t}(\omega)$ (at one local minimum). Then since local minima cannot recur $X_{\tau}(\omega)$ oscillates from the right at $\tau$ and thus $\tau$ is a limit point from the right of points in $\mathscr{T}_{j}(\omega)$. So $\mathscr{T}_{j}(\omega)$ is perfect. Define $T_{j}(\omega)=\mathscr{T}_{j}(\omega) \cap Q_{4}\left[X_{t}(\omega)\right]$. Now $\mathscr{T}_{j}(\omega)$ is a perfect set of measure zero. Also $T_{j}(\omega)$ was formed by removing $\boldsymbol{\aleph}_{0}$ points from $\mathscr{T}_{j}(\omega)$ and so $T_{j}(\omega)$ is a nowhere dense $G_{\delta}$ condensing at zero. We see that $Q_{4}\left[X_{t}(\omega)\right]$ is a $G_{\delta \sigma}$ of first category condensing at each real number.

We can now conclude that almost surely $Q_{1}\left[X_{t}(\omega)\right]$ is a $G_{\delta}$ since in the above paragraph its complement was shown to be the union of four sets of the form $\bigcup_{j=0}^{\infty} \mathscr{T}_{j}(\omega)$ each of which are $F_{\sigma}$ 's. This completes the proof of the theorem.

As mentioned previously [1] gives the remarkable result that almost surely a sample path is devoid of points of increase and decrease. The weaker statement that almost surely a sample path is devoid of points of improper increase and improper decrease is an immediate consequence of Shepp's Lemma. The relative strength of the role played by points of maxima as compared to the null role of points of increase is further emphasized by noting that almost every sample path is completely determined by its maxima. This follows from the almost sure denseness of the maxima and the continuity of the sample paths.

A function $f(t)$ is said to be locally recurrent at $t$ if there exists a sequence of distinct points $t_{n}$ converging to $t$ such that $f\left(t_{n}\right)=f(t)$. We shall modify the Wiener process sample paths to obtain continuous, nonconstant sample paths which almost surely are locally recurrent everywhere with a dense set of points of differentiability. Until recently the question of whether such a function could exist was an unsolved problem [4].

Suppressing $\omega$ we let $g(t)$ denote any Wiener process path for which the Theorem is valid. Define

$$
\begin{aligned}
& f_{0}(t)=0 \quad \text { if } t \in(-\infty, 0] \text {, } \\
& =g(t) \text { if } t \in[0,1] \text {, } \\
& =g(1) \text { if } t \in[1, \infty) \text {. }
\end{aligned}
$$

Notice that $f_{0}$ is locally recurrent except where $g$ has a proper maximum or 
minimum on $(0,1)$. Let $\left\{m_{i}\right\}$ be this set on $(0,1)$ and define $I(i)=\{j \mid j<i$ and $\left.m_{j}<m_{i}\right\}$. Define

$$
\begin{aligned}
f_{i}(t) & =f_{i-1}(t) & & \text { if } t \in\left(-\infty, \hat{m}_{i}\right], \\
& =f_{i-1}\left(\hat{m}_{i}\right) & & \text { if } t \in\left[\hat{m}_{i}, \hat{m}_{i}+\delta_{i}\right], \\
& =f_{i-1}\left(t-\delta_{i}\right) & & \text { if } t \in\left[\hat{m}_{i}+\delta_{i},+\infty\right),
\end{aligned}
$$

where $\hat{m}_{1}=m_{1}$ and $\hat{m}_{i}=m_{i}+\sum_{j \in I(i)} \delta_{j}$ for $i>1$. The number $\delta_{i}>0$ is chosen so that $\sup \left|f_{i}-f_{i-1}\right|<1 / 2^{i}$. It is possible to choose such a $\delta_{i}$ since $f_{i-1}$ is uniformly continuous. Thus given any $\varepsilon>0$ there exists a $\delta>0$ such that $\left|f_{i}(t)-f_{i}(y)\right|<\varepsilon$ whenever $|t-y|<\delta$. This implies that for $k>m$, sup $\left|f_{k}-f_{m}\right|<1 / 2^{m}$. We see that $f_{i}$ converges uniformly to a continuous function $f$.

It is clear that $f$ is nonconstant since it assumes the values 0 and $g(1)$. To see that $f$ is differentiable at a dense set of points observe that $\left\{m_{i}\right\}$ is dense in $(0,1)$. This implies that in every neighborhood there is an interval where $f$ is constant, for suppose not, then $f$ on this interval, $0_{1}$, equals $f_{0}$ on some interval, $0_{2}$, of $(0,1)$. But on $0_{2}$ must be some $m_{k}$. Thus $f$ must be constant on a subinterval of $0_{1}$.

Finally to show that $f$ is locally recurrent everywhere it is convenient to view the mapping from $f_{0}$ to $f$ as a sequence of stretchings of the $t$ axis where the point $\hat{m}_{j}$ becomes an interval of length $\delta_{j}$ while points to the left of it are unchanged and points to the right are translated by $\delta_{j}$ but maintain their original functional value. We shall correspond points before the stretching to points or intervals after $f$ is constructed. If the original points were in $(-\infty, 0]$ or $[1, \infty)$ they are transformed into points where $f$ is locally recurrent. If the original point was an $m_{i}$ its image after the stretching process is clearly an interval where $f$ is locally recurrent. It remains to show that if $t \in(0,1)$ and $t \notin\left\{m_{i}\right\}$ then $f$ is locally recurrent at $t$, the image of $t$ under the stretching. Look at an open interval containing $\hat{t}$. This interval must contain a point $y$ which is an image of an $m_{k}$ and a $\hat{y}$ which is an image of an $m_{l}$ such that $y<\hat{t}<\hat{y}$. Since $f_{0}$ is locally recurrent at $x$ there is a $w \neq \hat{t}$ which is an image of a $v$ where $m_{k} \leqq v \leqq m_{l}$ and $f_{0}(v)=f_{0}(t)$. Thus $y<w<\hat{y}$ and $f(w)=f(\hat{t})$.

An interesting consequence of this example is that it enables the decomposition of the real line into $c$ disjoint perfect sets. Evidently the inverse image of each point in the range of $f$ is a perfect set.

In light of what we have covered in this paper it is interesting to consider the following statement found in [5]. For any value a, the set $T^{(a)}=\left\{t \mid X_{t}(\omega)=a\right.$, $0 \leqq t \leqq 1\}$ is for almost all $\omega$ either empty or a perfect closed set of Lebesgue measure zero. A cursory analysis of this statement might lead one to the erroneous conclusion that almost surely the inverse image of every functional value of $X_{t}(\omega)$, $0 \leqq t \leqq 1$ is either empty or a perfect set.

There is a remarkable similarity between the local nature of the Wiener process sample paths and the local nature of the Weierstrass function. We refer the reader to Garg [6] for a discussion of the Weierstrass function.

AcKnOwLedgment. We wish to express our thanks to L. A. Shepp who stimulated our interest in [1]. 


\section{REFERENCES}

1. A. Dvoretzky, P. Erdös and S. Kakutani, Nonincrease everywhere of the Brownian motion process, Proc. Fourth Berkeley Sympos. Math. Statist. and Prob., vol. II, Univ. of California Press, Berkeley, Calif., 1961, pp. 103; 105; 107. MR 24 \#A2448.

2. J. L. Doob, Stochastic processes. Chapter 8, Wiley, New York, 1962. MR 15, 445.

3. J. Pierpont, Lectures on the theory of functions of real variables, Dover, New York, 1959, p. 524. MR 21 \#4208.

4. J. G. Mauldon, The differentiability of locally recurrent functions, Amer. Math. Monthly 72 (1965), 983-985. MR 32 \#4222.

5. L. Breiman, Probability, Addison-Wesley, Reading, Mass., 1968, p. 267. MR 37 \#4841.

6. K. M. Garg, On asymmetrical derivatives of nondifferentiable functions, Canad. J. Math. 20 (1968), 135-143. MR 36 \#3930.

Bell Telephone Laboratories, Inc.

HOLMDEL, NEW JERSEY 07733 